\title{
On Random SA-mixed Network Models Generated From Sierpinski and Apollonian networks
}

\author{
Jing $\mathrm{Su}^{1, \mathrm{a}}$, Bing $\mathrm{Yao}^{2, \mathrm{a}}$, Ming $\mathrm{Yao}^{3, \mathrm{~b}}$ \\ ${ }^{1}$ College of Mathematics and Statistics, Northwest Normal University, \\ Lanzhou, Gansu 730070, China \\ ${ }^{2}$ Department of Information Process and Control Engineering, Lanzhou, China \\ aemail: yybb918@163.com, bemail: yybm918@163.com
}

Keywords: Scale-free; Sierpinski networks; Apollonian networks; Degree cumulative distribution; Velocity.

\begin{abstract}
We constructed the random SA-mixed models based on Sierpinski and 2-dimension Apollonian networks having scale-free properties, and we have shown our random SA-mixed models are scale-free. It is noticeable, adding unchanged motifs doesn't affect the scale-free behaviors of the origin networks. The random SA-mixed models are scale-free by proofs of extremum methods.
\end{abstract}

\section{Introduction}

Many real-life networks can be described as scale-free network models. In recent years, with the rapid development of the Internet of Things [1][2], it shows the scale-free feature increasingly in metabolic networks, communication networks and scientific literature [3][4]. In order to explore the topological structures of real-world networks, the researchers have built a variety of scale-free models. Such as deterministic scale-free web and deterministic recursive graphs, Sierpinski networks [5] and Apollonian networks [6] etc.

Network motifs are an important local property of networks, and have been identified in a wide range of networks across many scientific disciplines and are suggested to the basic building blocks of most complex networks [7][8]. Motifs are of notable importance largely because they may reflect functional properties, and they have recently gathered much attention as a useful concept to answer structural design principles of scale-free networks, and may provide a deep insight into the network's functional abilities [9].

In this paper, we, in order to mimic real-world networks, build up $S A$-mixed models on the basis of Sierpinski networks and 2-dimension Apollonian networks by using the idea of motifs. We add motifs randomly in the $S A$-mixed models, therefore, it can simulate real networks better, and verify its scale-free by proving the scale-freeness of maximal model and minimal model respectively to approximate it. In addition, we prove the scale-freeness the velocities of Sierpinski networks, Apollonian networks and $S A$-mixed models. Our results show that the velocity of $S A$-mixed models is faster than other two networks. It is a guidance for us to build different networks according to the needs of human.

\section{SA-mixed models}

We define some fractal operations before we show our models, and some fractal operations can be found in [5]. Let $S(0)$ be a graph having three vertices $A, B, C$ and three edges such that any pair of vertices is joined by an edge, we call $S(0)$ a triangle (see Figure 1(a) and (b)). Clearly, the triangle $S(0)$ divides the plane into two parts: One is out of $S(0)$, called the outer face of $S(0)$; one is inside $S(0)$, called the inner face of $S(0)$.

A fractal (1)-operation yields a configuration $H_{(1)}$ (see Figure 1(c)) in the way: Add a vertex $u$ into the inner face of a triangle shown in Figure 1(a), and then join $u$ with three vertices $A, B$ and $C$ by three edges, respectively. We define a labelling function $g$ for $H_{(1)}$ as: $g(A)=g(B)=g(C)=k-1$, 
$g(u)=k$ for $k \geq 1$.

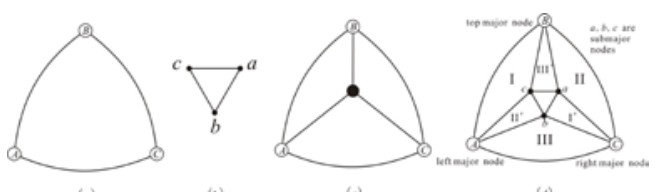

Figure1 Two fractal operations.

A fractal (II)-operation produces a complex configuration $H_{(2)}$ (see Figure $1(\mathrm{~d})$ ) in the way: Put an operation triangle $a b c$ shown in Figure 1(b) into the inner face of an objective triangle $A B C$ shown in Figure 1(a), and furthermore join $c$ with $A$ and $B$ by two edges, join $a$ with $B$ and $C$ by two edges, and then join $b$ with $C$ and $A$ by two edges. Clearly, $H_{(2)}$ has 7 inner faces and an outer face. We define a labelling function $f$ of $H_{(2)}$ as: $f(A)=f(B)=f(C)=k-1, f(a)=f(b)=f(c)=k$ for $k \geq 1$.

Sierpinski and Apollonian models.

Sierpinski model $S(t)$ The Sierpinski model $S(t)$ can be generated by the Sierpinski-algorithm1.

\section{Algorithm1 Sierpinski-algorithm}

Initialization. At time step $t=0, S(0)$ is shown in Figure 2, and the labelling function $f$ holds $f(A)=f(B)=f(C)=0$.

Iteration. At time step $t$, do a fractal (II)-operation to every objective triangle $x y z$ of $S(t-1)$ with no $f(x)=f(y)=f(z)$ and at least one of three labels $f(x), f(y)$ and $f(z)$ is equal to $t-1$, and label three vertices of each operation triangle with $t$ under $f$.

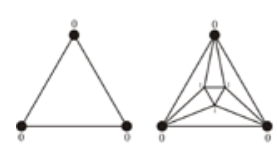

$s(0)$ $s(1)$

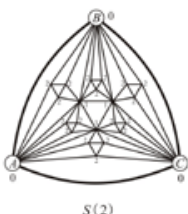

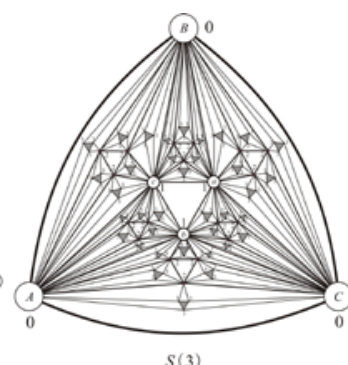

Figure2: The construction of the Sierpinski model S(t) at the first four steps.
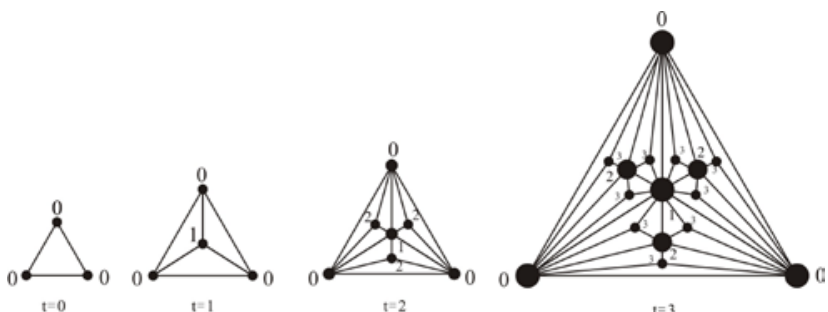

Figure3: The construction of the Apollonian networks at the first four steps.

The Sierpinski model $S(t)$ has its own vertex number $n_{v}^{s}(t)$ and edge number $n_{e}^{s}(t)$, and we have $n_{v}^{s}(t)=\frac{3 \cdot 6^{t}+12}{5}$ and $n_{e}^{s}(t)=\frac{9 \cdot 6^{t}+6}{5}$ at time step $t$. So for large $t, S(t)$ has its own average degree $\langle\mathrm{k}\rangle=\frac{2 n_{e}^{S}(t)}{n_{v}^{S}(t)}$ that is approximate to 6. And the cumulative distribution $P_{\text {cum }}(k)$ of $S(t)$ is $P_{\text {cum }}(k)=6(k-1)^{1-\gamma_{s}}$ with $\gamma_{s}=2+\frac{\ln 2}{\ln 3}$, thus, $S(t)$ is scale-free [5].

Apollonian model $A(t)$ The Apollonian model $A(t)$ can be generated by the Apollonian-algorithm 2.

\section{Algorithm2 Apollonian-algorithm}

Initialization. At time step $t=0, A(0)$ is a triangle $A B C$, and the labelling function $f$ holds $f(A)=f(B)=f(C)=0$.

Iteration. At time step $t$, do a fractal (I)-operation to every triangle $x y z$ of $A(t-1)$ with at least the number of three labels $f(x), f(y)$ and $f(z)$ is equal to $t-1$, and label the vertex added with $t$ under $f$.

See $A(0), A(1), A(2)$ and $A(3)$ shown in figure-3. The Apollonian model $A(t)$ has its own properties as [6]: At time step $t$, the number of vertices of $A(t)$ is $n_{v}^{A}(t)=\frac{3^{t}+5}{2}$, and the number of edges of $A(t)$ is $n_{e}^{A}(t)=\frac{3^{t+1}+3}{2}$, the average degree of $A(t)$ is $\langle k\rangle=\frac{2 n_{e}^{A}(t)}{n_{v}^{A}(t)}=\frac{6\left(3^{t}+1\right)}{3^{t}+5} \rightarrow 6$ for large $t$. 
The cumulative distribution of $A(t)$ is $P_{\text {cum }}(k) \sim \frac{1}{2} \cdot 3^{1-\frac{\ln 3}{\ln 2}} \cdot k^{1-\gamma_{A}}$ with $\gamma_{A}=1+\frac{\ln 3}{\ln 2}$. When the size of the Apollonian model $A(t)$ is large, the degree distribution $P(k)$ follows a power law with the exponent between 2 and 3. Therefore, the Apollonian model $A(t)$ is also scale-free.

\section{SA-mixed models.}

We are ready for constructing our $S A$-mixed models.

1) $S$ A-mixed model $S\left(t,\left.A\left(m_{i}\right)\right|_{1} ^{n}\right)$

An $S A$-mixed model $S\left(t, A\left(m_{1}\right), A\left(m_{2}\right), \ldots, A\left(m_{n}\right)\right)$ can be built up by the $S A$-mixed-algorithm 3 . For $\mathrm{n}$ Apollonian models $A\left(m_{1}\right), A\left(m_{2}\right), \ldots, A\left(m_{n}\right)$, we arrange the vertex numbers of $A\left(m_{i}\right)$ with $i=1,2, \ldots, n$ in an ordered order from small to large, in other words, the vertex number of $A\left(m_{j}\right)$ does not exceed that of $A\left(m_{j+1}\right)$ for $j=1,2, \ldots n-1$, we call them motifs.

\section{Algorithm3 SA-mixed-algorithm \\ Initialization. At time step $t_{0}, S\left(t_{0},\left.A\left(m_{i}\right)\right|_{1} ^{n}\right)$ is obtained by choose randomly $A\left(m_{1}\right)$, $A\left(m_{2}\right), \ldots, A\left(m_{n}\right)$ and put randomly them into $6^{t_{0}-1}$ objective triangles in which every triangle $A B C$ holds $f(A)=f(B)=f(C)=t_{0}$. \\ Iteration. At time step $t$, do a fractal (III)-operation to every objective triangle $A B C$ of $S(t)$. The resulting model is just an SA-mixed model $S\left(t, A\left(m_{1}\right), A\left(m_{2}\right), \ldots, A\left(m_{n}\right)\right)$.}

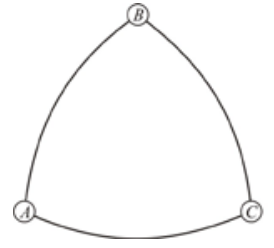

Figure4: The fractal (III)-operation.

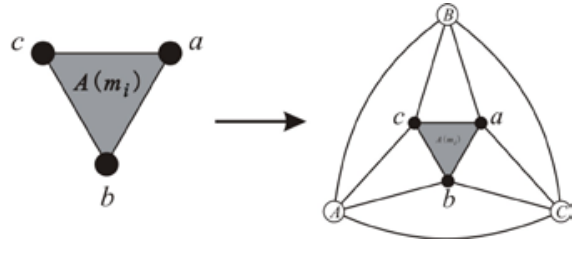

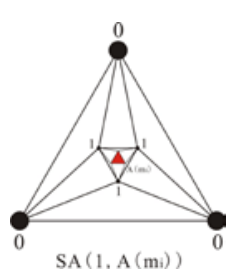

Figure5: The construction of the SA-mixed models at the time step 1.

Before realizing our goal, we define the fractal (III)-operation that is a mixed operation of the fractal (I)-operation and the fractal (II)-operation. We label the three vertices of the outer face of an Apollonian model $A\left(m_{i}\right)$ with $a, b, c$, and put this $A\left(m_{i}\right)$ into the inner face of an objective triangle $A B C$, and do a fractal (II)-operation (see Figure 4). Since the Sierpinski model $S(j)$ has $6^{j-1}$ objective triangle $A B C$ with $f(A)=f(B)=f(C)=j$ at time step $j$, we can set the initial SA-mixed model $S\left(t_{0},\left.A\left(m_{i}\right)\right|_{1} ^{n}\right)$ by $6^{t_{0}-1} \geq n$.

We write $S\left(t,\left.A\left(m_{i}\right)\right|_{1} ^{n}\right)$ instead of $S\left(t, A\left(m_{1}\right), A\left(m_{2}\right), \ldots, A\left(m_{n}\right)\right)$ for short. Because the number of all objective triangle $A B C$ with labeling $f(A)=f(B)=f(C)=t$ is $6^{t-1}$, so, the total number of the motifs $A\left(m_{i}\right)$ is also $6^{t-1}$. Notice that $6^{t-1}$ motifs $A\left(m_{i}\right)$ were chosen randomly and were settled randomly into $6^{t-1}$ objective triangles of $S(t)$. Therefore, it is difficult to compute the numbers of vertices and edges of $S\left(t,\left.A\left(m_{i}\right)\right|_{1} ^{n}\right)$, and then it is impossible to write the degree spectrum of $S\left(t,\left.A\left(m_{i}\right)\right|_{1} ^{n}\right)$.

If all motifs $A\left(m_{i}\right)$ added in implementing fractal (III)-operations to all objective triangles of $S(t)$ are equal to $A\left(m_{1}\right)$ at time step $t$, we get a particular $S A$-mixed model $S_{\min }\left(t,\left.A\left(m_{1}\right)\right|_{1} ^{n}\right)$. Similarly, another particular $S A$-mixed model $S_{\max }\left(t,\left.A\left(m_{n}\right)\right|_{1} ^{n}\right)$ can be obtained when all motifs $A\left(m_{i}\right)$ added in implementing fractal (III)-operations to all objective triangles of $S(t)$ are equal to $A\left(m_{n}\right)$.

In order to simplify the calculation, we let $n_{v}(t)$ and $n_{e}(t)$ be the total numbers of vertices and edges of $S\left(t,\left.A\left(m_{i}\right)\right|_{1} ^{n}\right)$ at step time $t$, respectively; and let $n_{v}^{\min }(t)$ and $n_{e}^{\min }(t)$ be the total numbers of vertices and edges of $S_{\min }\left(t,\left.A\left(m_{1}\right)\right|_{1} ^{n}\right)$ at step time $t$, respectively; and let $n_{v}^{\max }(t)$ and $n_{e}^{\max }(t)$ be the total numbers of vertices and edges of $S_{\max }\left(t,\left.A\left(m_{n}\right)\right|_{1} ^{n}\right)$ at step time $t$, respectively. Obviously, we get $\quad n_{v}^{\min }(t) \leq n_{v}(t) \leq n_{v}^{\max }(t) \quad, \quad n_{e}^{\min }(t) \leq n_{e}(t) \leq n_{e}^{\max }(t) \quad$.Thereby, we have 
$n_{v}^{\min }(t)=n_{v}^{S}(t)+6^{t-1} \cdot n_{v}^{A}\left(m_{1}\right)=\frac{6^{t-1}\left(5 \cdot 3^{m_{1}}+61\right)+24}{10} n_{e}^{\min }(t)=n_{e}^{S}(t)+6^{t-1} \cdot n_{e}^{A}\left(m_{1}\right)=\frac{6^{t-1}\left(5 \cdot 3^{m_{1}+1}+123\right)+12}{10}$

The average vertex degree $\langle k\rangle_{\min }$ of $S_{\min }\left(t,\left.A\left(m_{1}\right)\right|_{1} ^{n}\right)$ is equal to $\frac{2 n_{e}^{\min }(t)}{n_{v}^{\min }(t)} \rightarrow 6$ as $t \rightarrow \infty$. It shows $S_{\min }\left(t,\left.A\left(m_{1}\right)\right|_{1} ^{n}\right) \quad$ to $\quad$ be $\quad$ sparse. $\quad$ By $\quad n_{v}^{\max }(t)=n_{v}^{S}(t)+6^{t-1} \cdot n_{v}^{A}\left(m_{n}\right)=\frac{6^{t-1}\left(5 \cdot 3^{m_{n}}+61\right)+24}{10}$ and $n_{e}^{\max }(t)=\frac{6^{t-1}\left(5 \cdot 3^{m_{n}+1}+123\right)+12}{10}$, the average vertex degree $\langle k\rangle_{\max }$ of $S_{\max }\left(t,\left.A\left(m_{n}\right)\right|_{1} ^{n}\right)$ is equal to $\frac{2 n_{e}^{\max }(t)}{n_{v}^{\max }(t)} \rightarrow 6$ as $t \rightarrow \infty$, which means that $S_{\max }\left(t,\left.A\left(m_{n}\right)\right|_{1} ^{n}\right)$ is also sparse. We can guess $: "$ the $S A$-mixed model $S\left(t,\left.A\left(m_{i}\right)\right|_{1} ^{n}\right)$ is sparse". Furthermore, $S_{\max }\left(t,\left.A\left(m_{n}\right)\right|_{1} ^{n}\right)$ its own maximum degree does not exceed $3 \cdot 2^{m_{n}-1}+3^{t}-1$ and its minimum degree is 3 .

\section{2) Degree cumulative distribution of the $S A$-mixed models}

To show our models scale-free nature, in the following we concentrate on the degree cumulative distribution $P_{\text {cum }}(k)$ to measure the degree distribution $P(k)$ of the $S A$-mixed model $S\left(t,\left.A\left(m_{i}\right)\right|_{1} ^{n}\right)$, where the degree distribution $P(k)$ is the probability that a randomly selected vertex has exactly $k$ edges in $S\left(t,\left.A\left(m_{i}\right)\right|_{1} ^{n}\right)$.

For estimating the topological structure of $S\left(t,\left.A\left(m_{i}\right)\right|_{1} ^{n}\right)$, we compute the degree cumulative distributions $P_{\text {cum }}^{\min }(k), P_{\text {cum }}^{\max }(k)$ and the degree distributions $P^{\min }(k), P^{\max }(k)$ of two $S A$-mixed models $S_{\min }\left(t,\left.A\left(m_{1}\right)\right|_{1} ^{n}\right)$ and $S_{\max }\left(t,\left.A\left(m_{n}\right)\right|_{1} ^{n}\right)$, respectively, in the following argument. Since the degree spectrums of two models $S_{\min }\left(t,\left.A\left(m_{1}\right)\right|_{1} ^{n}\right)$ and $S_{\max }\left(t,\left.A\left(m_{n}\right)\right|_{1} ^{n}\right)$ are discrete, so we can use a technique introduced by Newman in [11],[12], Dorogovstev defined the cumulative distribution $P_{\text {cum }}(k)=\sum_{k^{\prime} \geq k} \frac{\left|V\left(k^{\prime}, t\right)\right|}{n_{v}(t)} \propto k^{1-\lambda}$, where $\left|\mathrm{V}\left(k^{\prime}, t\right)\right|$ is the number of vertices of degree $k^{\prime}$ [12]. Here, we get the cumulative distribution of $S_{\min }\left(t,\left.A\left(m_{1}\right)\right|_{1} ^{n}\right)$ as follows $P_{\text {cum }}^{\min }(k)=\sum_{k^{\prime} \geq k} \frac{\left|V\left(k^{\prime}, t\right)\right|}{n_{v}(t)}=\frac{36 \cdot 6^{\tau-1}+5 \cdot 3^{m_{1}}}{6^{t-1}\left(5 \cdot 3^{m_{1}+1}+123\right)}$, where $\tau$ is the time that vertices with degree $k$ added in the $S A$-mixed model, plugging $\tau=t+2-\frac{1}{\ln 3} \ln \left(k-2^{m_{1}+1}+2^{m_{1}}\right)$ into the above equation, and when $t$ is large enough, we can obtain $P_{c u m}^{\min }(k)=\frac{1290}{5 \cdot 3^{m_{1}+1}+123} \cdot\left(k-2^{m_{1}+1}+2^{m_{1}}\right)^{-\left(1+\frac{\ln 2}{\ln 3}\right)}$.According to [12] it is convenient to obtain that the degree distribution via its cumulative degree distribution $P^{\min }(k)=-\frac{\partial P_{c u m}^{\min }(k)}{\partial k}=\frac{1290}{5 \cdot 3^{m_{1}+1}+123}\left(1+\frac{\ln 2}{\ln 3}\right)\left(k-2^{m_{1}+1}+2^{m_{1}}\right)^{-\left(2+\frac{\ln 2}{\ln 3}\right)}$, Thereby, the degree distribution $P^{\min }(k)$ follows a power law form with the exponent $\gamma=2+\frac{\ln 2}{\ln 3}$, where $2<\gamma<3$. It indicates that the minimal model of random $S A$-mixed models is scale-free.

For the model $S_{\max }\left(t,\left.A\left(m_{n}\right)\right|_{1} ^{n}\right)$, we have $P_{c u m}^{\max }(k)=\sum_{k^{\prime} \geq k} \frac{\left|V\left(k^{\prime}, t\right)\right|}{n_{v}(t)}=\frac{36 \cdot 6^{\tau-1}+5 \cdot 3^{m_{n}}}{6^{t-1}\left(5 \cdot 3^{m_{n}+1}+123\right)}$ plugging $\tau=t+2-\frac{1}{\ln 3} \ln \left(k-2^{m_{n}+1}+2^{m_{n}}\right)$ into the above equation, and when $t$ is large enough, we can obtain $P_{\text {cum }}^{\max }(k)=\frac{1290}{5 \cdot 3^{m_{n}+1}+123} \cdot\left(k-2^{m_{n}+1}+2^{m_{n}}\right)^{-\left(1+\frac{\ln 2}{\ln 3}\right)}$ Similarly, with $P^{\max }(k)=-\frac{\partial P_{c u m}^{\max }(k)}{\partial k}$, we can easily compute the degree distribution $P^{\max }(k) \propto C k^{-\gamma}, 2<\gamma=2+\frac{\ln 2}{\ln 3}<3$. where $C$ is a constant. So, the 
maximal model of random SA-mixed models is also scale-free.

Due to the degree distribution of the maximum model and the minimum model are all obey power law distribution, we can use this two models to approximate the random $S A$-mixed models. Obviously, we have $P^{\min }(k) \leq P(k) \leq P^{\max }(k)$, which means that the random $S A$-mixed models also follows certain power law distributions, so they are scale-free.

\section{3) Velocities of the models}

The dynamic development speed of networks was proposed by [13], this metric is used to measure the speed development of real networks. The velocity of the minimum random $S A$-mixed model

$$
S_{\min }\left(t,\left.A\left(m_{1}\right)\right|_{1} ^{n}\right)
$$

can be computed

as

follows $\quad V_{e l}^{\min }(t)=\frac{\partial n_{v}^{\min }(t)}{\partial t} \cdot \frac{\partial n_{e}^{\min }(t)}{\partial t}=\frac{\left(5 \cdot 3^{m_{1}}+61\right)\left(5 \cdot 3^{m_{1}+1}+123\right)}{3600}\left(6^{t} \ln 6\right)^{2} \propto \frac{3^{2 m_{1}}}{124}\left(6^{t} \ln 6\right)^{2} \quad$.For the maximum random $S A$-mixed model $S_{\max }\left(t,\left.A\left(m_{n}\right)\right|_{1} ^{n}\right)$, it's velocity is $V_{e l}^{\max }(t)=\frac{\partial n_{v}^{\max }(t)}{\partial t} \cdot \frac{\partial n_{e}^{\max }(t)}{\partial t}=\frac{\left(5 \cdot 3^{m_{n}}+61\right)\left(5 \cdot 3^{m_{n}+1}+123\right)}{3600}\left(6^{t} \ln 6\right)^{2} \propto \frac{3^{2 m_{n}}}{124}\left(6^{t} \ln 6\right)^{2}$. On the other hands, we have the velocity of networks as $V_{e l}(S(t))=\frac{\partial n_{v}^{S}(t)}{\partial t} \cdot \frac{\partial n_{e}^{S}(t)}{\partial t}=\frac{3}{5} 6^{t} \ln 6 \cdot \frac{9}{5} 6^{t} \ln 6=\frac{27}{25}\left(6^{t} \ln 6\right)^{2}$ and the velocity of Apollonian networks as $V_{e l}(A(t))=\frac{\partial n_{v}^{A}(t)}{\partial t} \cdot \frac{\partial n_{e}^{A}(t)}{\partial t}=\frac{1}{2} 3^{t} \ln 3 \cdot \frac{3}{2} 3^{t} \ln 3=\frac{3}{4}\left(3^{t} \ln 3\right)^{2}$.Combine the above velocities together in the way $V_{e l}\left(S_{\min }\left(t,\left.A(0)\right|_{1} ^{n}\right)\right)<V_{e l}\left(S\left(t,\left.A\left(m_{i}\right)\right|_{1} ^{n}\right)\right)<V_{e l}\left(S_{\max }\left(t,\left.A\left(m_{n}\right)\right|_{1} ^{n}\right)\right)$.And we have $V_{e l}\left(S\left(t,\left.A\left(m_{i}\right)\right|_{1} ^{n}\right)\right)>V_{e l}(S(t))$, contrast to $V_{e l}(A(m)), V_{e l}\left(S\left(t,\left.A\left(m_{i}\right)\right|_{1} ^{n}\right)\right)>V_{e l}(A(m))$. Moreover, we have $\left|V_{e l}^{\max }(t)-V_{e l}^{\min }(t)\right| \approx \frac{\left(6^{t} \ln 6\right)^{2}}{124}\left(3^{2 m_{n}}-3^{2 m_{1}}\right)=\frac{\left(6^{t} \ln 6\right)^{2}}{124 \cdot 3^{2 m_{1}}}\left[3^{2\left(m_{n}-m_{1}\right)}-1\right] \quad$.For $\quad\left|m_{n}-m_{1}\right| \quad<\varepsilon$, then $\left|V_{e l}^{\max }(t)-V_{e l}^{\min }(t)\right|<\varepsilon$ with $\varepsilon>0$. Hence, we can control $V_{e l}(t)$ by computing $V_{e l}^{\min }(t)$ and $V_{e l}^{\max }(t)$. It can simulate the real-network because of its randomness. This $S A$-mixed model can satisfy higher requirements on the speed of network, such as we can curb the speed of rumors and prevent the speed of infectious diseases.

\section{More network models of SA-mixed models.}

For further researching $S A$-mixed models, we are considering the following possible researching problems: - Add $n$ Apollonian networks $A\left(m_{1}\right), A\left(m_{2}\right), \ldots, A\left(m_{n}\right)$ to every triangle $\triangle A B C$ of Sierpinski network at time step $t$ with labeling $f(A) \neq f(B), f(B) \neq f(C)$ and $f(A) \neq f(C)$. We call this network DL (different label) $S A$-mixed models. $\bullet$ Add $n$ Apollonian networks $A\left(m_{i}\right)(i=1,2, \ldots, n)$ to $\left\lceil P \cdot 6^{t-1}\right\rceil$ triangles $\triangle A B C$ of Sierpinski networks at time step $t$ with labeling $f(A)=f(B)=f(C)$, where $P$ is market regulative parameters and $0 \leq P \leq 1$. We refer to this network MRSL (market regulation some label) random $S A$-mixed model. When $P=1$, MRSL random $S A$-mixed model is one introduced in this paper. Similarly, if we add $A\left(m_{i}\right)(i=1,2, \ldots, n)$ to $\left\lceil P \cdot n_{\Delta}(t)\right\rceil$ triangles with $f(A) \neq f(B), f(B) \neq f(C)$ and $f(A) \neq f(C), 0 \leq P \leq 1$, where $n_{\Delta}(t)$ is the total number of $\triangle A B C$ for $n_{\Delta}(t)=12$ with $t=2$, and $n_{\Delta}(t)=84 \cdot 6^{t-3}$ with $t \geq 3$. $\bullet$ n the basis of random SA-mixed models, we use a graph $G$ with three vertices at least a triangle to replace 2-dimension Apollonian networks $A\left(m_{i}\right)$ with $i=1,2, \ldots, n$ and regard $G$ as a motif, the resulting models are called $S G$-mixed models.

\section{Conclusion}

In this paper, the $S A$-mixed models which we study in this paper are constructed by mixing randomly which have scale-free characteristics. We believe that our models may help engineers in network topology-designing and performance analyzing, it also can help to regulate markets. Clearly, the models which we proposed are maximum planar graph, so it might help to understand some properties of real-world planar networks and may be helpful for designing printed circuits. In 
a similar way, we change models from Apollonian networks to any graph $G$ with three vertices at least a triangle. We have shown that they have scale-free characteristics, which display similar features. The networks present the typical characteristics if real-life networks in nature and society as they have a power-law degree distribution. We compare them by computing analytical expressions for the degree distribution and their velocity.

\section{Acknowledgement}

The authors are grateful to the anonymous referees for their valuable comments and suggestions. The second author, Bing Yao, thanks the National Natural Science Foundation of China under grants (Project No. 61163054 and No. 61363060) The third author, Ming Yao, thanks The Special Funds of Finance Department of Gansu Province of China under grant (Project No. 2014-63).

\section{References}

[1] Bing Yao, Xia Liu, Wanjia Zhang, Xiang'en Chen, Xiaomin Zhang, Ming Yao, Zheng-xue Zhao. Applying Graph Theory To The Internet Computing and Communications and 2013 IEEE International Conference in Embedded and Ubiquitous Computing, 2354-2361. DOI:10.1109/HPCC.and.EUC.2013.339.

[2] Bing Yao, Chao Yang, Ming Yao, Hongyu Wang, Xiang'en Chen, Xiaomin Zhang, Mogang Li. Graphs As Models of Scale-free Networks. Applied Mechanics and Materials, Vol.380-384(2013) pp 2034-2037. DOI: 10.4028/www.scientific.net/AMM.380-384.2034

[3] Albert, R., Jeong, H., and Barabasi, A.-L., Nature, 401,130(1999).

[4] Z.Z.Zhang, S.G.Zhou, T.Zou, L.C.Chen, and J.H.Guan, Eur. Phys. J.B60, 259(2007).

[5] Zhongzhi Zhang, Shuigeng Zhou, Lujiu Fang, Jihong Guan and Yichao Zhang. Maximal planar scale-free Sierpinski networks with small-world effect and power-law strength-degree correlation. EPL(Europhysics Letters) 2007, 79: 38007.

[6] Zhongzhi Zhang, Francesc Comellas, Guillaume Fertin and Lili Rong. High dimensional Apollonian networks. E-print cond-mat/0503316.

[7] Rui Jiang, Zhidong Tu, Ting Chen and Fengzhu Sun. Network motif identification in stochastic networks. PNAS June 20, 2006 vol. 103 no. 25, 9404C9409.

[8] Radu Dobrin, Qasim K Beg, Albert-Laszlo Barabasi and Zoltan N Oltvai. Aggregation of topological motifs in the Escherichia coli transcriptional regulatory network. BMC Bioinformatics 2004, 5: 10 .

[9] Masoudi-Nejad A, Schreiber F, Razaghi MK Z. Building Blocks of Biological Networks: A Review on Major Network Motif Discovery Algorithms. IET Syst Biol Volume: 6 Issue: 164-74, 2012.

[10] Barabasi, A.-L., and Albert, R., Science, 286,509(1999).

[11] M.E.J.Newman, The structure and function of complex networks, SIAM Review 45(2003) 167-256.

[12]S.N.Dorogovstev, A.V. Goltsev, J.F.F. Mendes. Pseufractal scale-free web. Physiacal review 2002, 65, 066122-066125.

[13]Bing Yao, Xiaomin Wang, Jing Su, Fei Ma, Ming Yao, Mingjun Zhang, and Jianmin. Methods And Problems Attempt in Scale-Free Models From Complex Networks Xie. submitted 